\title{
A NEW CONFIGURATION OF IRREGULAR REFLECTION OF SHOCK WAVES
}

\author{
L. G. Gvozdeva and S. A. Gavrenkov \\ Joint Institute for High Temperatures \\ Russian Academy of Sciences \\ 13-2 Izhorskaya Str., Moscow 125412, Russia
}

\begin{abstract}
A new configuration of shock waves has been found in the reflection of shock waves in a stationary supersonic gas flow in addition to the wellknown regular and Mach reflections. This new three-shock configuration occurs with a negative angle of reflection and Mach numbers greater than 3 and an adiabatic index smaller than 1.4. It has been shown that this new configuration is unstable and leads to a radical change of the total flow pattern. The emergence of this new kind of instability can negatively affect operation of aircraft and rocket engines due to the failure of the flow to be as conventionally predicted.
\end{abstract}

\section{INTRODUCTION}

The reflection of shock waves is a fundamental element of external and internal aerodynamics $[1,2]$. In a stationary supersonic stream of gas, two types of reflections are known: the two-shock (regular reflection) and the three-shock wave configurations (irregular or Mach reflection). One can see these, for example, at the entrance of an aircraft air inlet (Figs. $1 a$ and $1 b$ ) or at the overexpanded jet from a rocket engine nozzle (Fig. 1c). In this work, the three-shock configuration is considered; it consists of a incident wave $I A$, a reflected wave $A R$ and a Mach stem $A M$. These waves intersect in a triple point $A$. The arrangement of waves is defined by the Mach number of flow $\mathrm{M}_{1}$, the angle of incidence $\omega_{1}$, and the effective adiabatic index of gas $\gamma$.

Typically, the predicted and observed flow pattern looks as in Figs. $1 b$ and $1 c$, i. e., the reflected wave $A R$ is located above the line of incoming flow. This configuration will be called a configuration with a positive angle of reflection $\left(\omega_{2}>0\right)$. Gvozdeva [3] has hypothesized that in a steady supersonic flow of gas at high Mach numbers and small values of the adiabatic index $\gamma$, the reflected wave must be located below the direction of inflow stream $\left(\omega_{2}<0\right)$; this constitutes

This is an Open Access article distributed under the terms of the Creative Commons Attribution License 4.0, which permits unrestricted use, distribution, and reproduction in any medium, provided the original work is properly cited. 


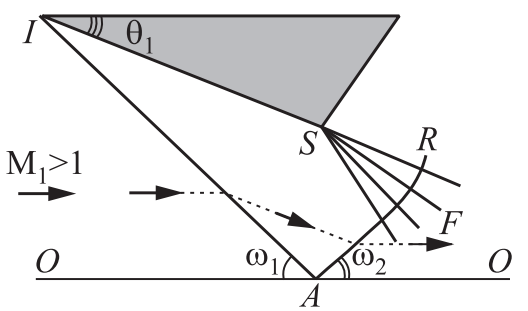

(a)

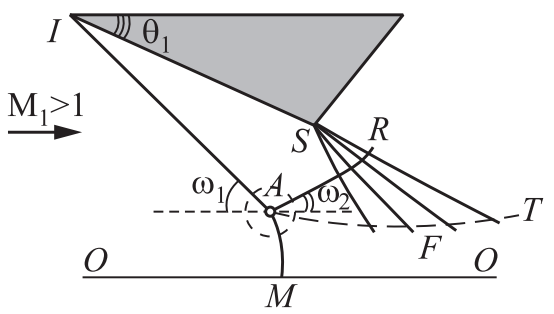

(b)

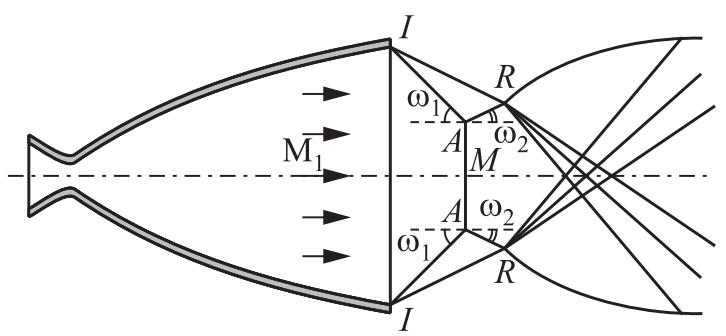

(c)

Figure 1 Two-shock $(a)$ and three-shock $(b)$ wave configurations at the entrance of an air intake and three-shock wave configuration at the outlet of the nozzle $(c): I A-$ incident shock; $A R$ - reflected shock; $A T$ - slip stream; $A M$ - Mach stem; $S F-$ rarefaction fan; $\omega_{1}$ - incidence angle; $\omega_{2}$ - reflection angle; $\theta_{1}$ - angle of the wedge; $A$ - triple point; $0-0$ - line of symmetry; and $\mathrm{M}_{1}$ - Mach number of the flow

a new configuration with a negative angle of reflection (Fig. 2). This configuration would be unstable and its appearance would lead to a radical change in the entire flow pattern. For example, let consider the case in Fig. $2 a$. If the reflected wave, which is going at the negative reflection angle $\omega_{2}$, were to intersect the line of symmetry $O O$, then the gas would start to accumulate in the closed area $A R M$. This would lead to choking of the flow and to the movement of the Mach stem upstream. So, there would be a radical change in the entire flow pattern. A similar process would occur in the overexpanded jet if the angle of reflection becomes negative (Fig. 2b). This idea was developed in [4-9] where preliminary analyses and testing of the calculation program have been presented.

In the present work, two questions have been answered:

1. Under what initial and boundary conditions could the new configuration exist?

2. What restructuring of the flow might result from the emergence of this new configuration? 


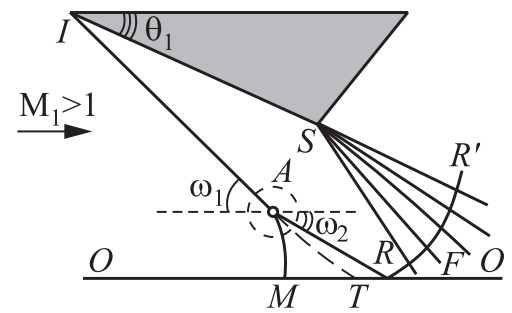

(a)

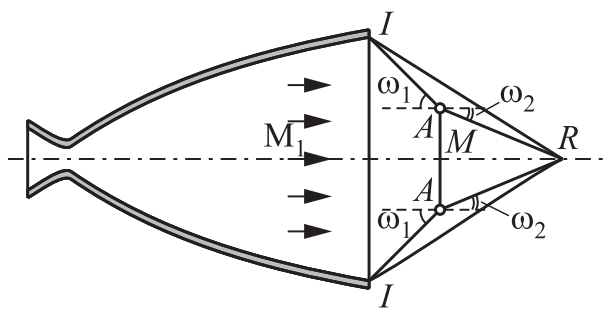

(b)

Figure 2 Three-shock wave configuration with a negative reflection angle $\omega_{2}<0$ at the inlet of the air intake $(a)$ and in overexpanded jet $(b)$. The notations are the same as in Fig. 1

\section{ANALYTICAL STUDIES}

\subsection{Analytical Calculations of the Boundaries of the Existence of the Three-Shock Configuration with a Negative Angle of Reflection}

To answer the first question, the analytical calculations have been done in accordance with the three-shock wave theory [3-11].

Let consider the steady supersonic flow of gas with adiabatic index $\gamma$, moving with a Mach number $\mathrm{M}_{1}$ (Fig. 3). The incident wave $I A$ is located at an angle to the incident flow $\omega_{1}$. The reflected wave $A R$ is located at an angle $\omega_{2}$, and a Mach stem $A M$ located at an angle $\omega_{3}$. It is assumed that there exists a certain neighborhood of the triple point where all three waves are straight. The three-shock theory suggests that the gas flow behind the incident wave is deflected by an angle $\theta_{1}$ and the gas behind the reflected wave is deflected in the opposite direction at a smaller angle $\theta_{2}$. In the Mach stem, gas is deflected by an angle $\theta_{3}$. The flow behind the incident and reflected waves must be parallel to the flow passing through the Mach stem and pressures on the tangential surface $A T$ must be the same. So, the compatibility conditions can be written as follows: $\theta_{3}=\theta_{1}-\theta_{2}$;

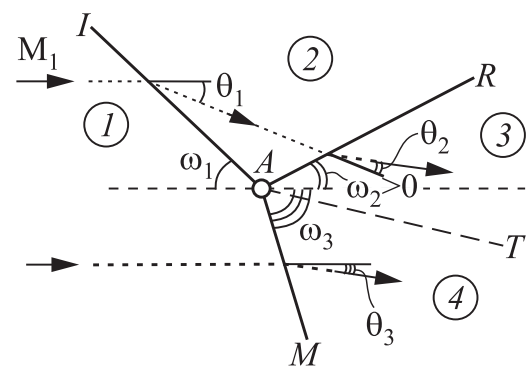

Figure 3 The location of shock waves in the area of triple point $A$ $p_{3}=p_{4}$. 
For each wave, one can write the system of equations:

$$
\begin{aligned}
\rho_{1} u_{1} & =\rho_{2} u_{2} ; & h & =C_{p} T ; \\
\rho_{1} u_{1}^{2}+p_{1} & =\rho_{2} u_{2}^{2}+p_{2} ; & C_{p} & =\frac{\gamma}{\gamma-1} \frac{R}{\mu} ; \\
\frac{u_{1}^{2}}{2}+h_{1} & =\frac{u_{2}^{2}}{2}+h_{2} ; & \mu & =\text { const } ; \\
p \mu & =\rho R T ; & \gamma & =\text { const }
\end{aligned}
$$

where $\rho$ is the density; $u$ is the velocity component normal to the shock wave; $p$ is the pressure; $h$ is the enthalpy; $\mu$ is the molecular weight; $R$ is the universal gas constant; $T$ is the temperature; $C_{p}$ is the specific heat at constant pressure; and $\gamma$ is the ratio of specific heats. Indices 1 and 2 correspond to the regions before and after the shock wave, respectively.

The relation of the angles in an oblique shock wave:

$$
\tan (\theta)=\frac{2}{\tan (\omega)} \frac{\mathrm{M}^{2} \sin ^{2} \omega-1}{\mathrm{M}^{2}(\gamma+\cos (2 \omega))+2}
$$

where $\omega$ is the angle between the wave and the flow line; $\theta$ is the angle of deflection of the flow passing through the shock wave; and $\mathrm{M}$ is the Mach number of the flow.

Solving this system of equations for a given $\mathrm{M}_{1}, \gamma$, and $\omega_{1}$, one can completely calculate the parameters of the flow behind the shock waves and determine the angles in the configuration. Calculations based on the three-shock theory give good agreement with experimental data for the strong shock waves when the flow of gas behind the reflected wave $A R$ is supersonic $[12,13]$. It should be noted that this theory determines only the angles and the parameters of the flow behind the shock wave near the triple point but does not determine the height of the Mach stem $A M$. Since the flow behind the Mach stem is subsonic, the height of it is determined by the geometry of the flow and conditions downstream.

The calculations have been performed in a wide range of initial parameters of Mach number $\mathrm{M}_{1}$ from 1 to 15 . The effective adiabatic index varied from 1.05 to 1.66 , the initial angle $\omega_{1}$ from $0^{\circ}$ to $90^{\circ}$. The results are presented as a function of the angle of reflection angle $\omega_{2}$ on the angle of incidence $\omega_{1}$ for different values of the Mach number $\mathrm{M}_{1}$ and the adiabatic index $\gamma$.

Figure $4 a$ shows that at constant adiabatic index $\gamma=1.4$, the curves $\omega_{2}$ $=f\left(\omega_{1}\right)$ lie only in the positive area for all values of the Mach number $\mathrm{M}_{1}$. The same is obtained for larger values of the adiabatic index $\gamma>1.4$. This explains the fact that all known three-shock-wave configurations are displayed only with a positive angle of reflection, because all experiments were conducted in wind tunnels in which the working gas is air with an adiabatic index $\gamma=1.4$. When reducing the adiabatic index, for example, down to $\gamma=1.3$ (Fig. $4 b$ ), the curves 


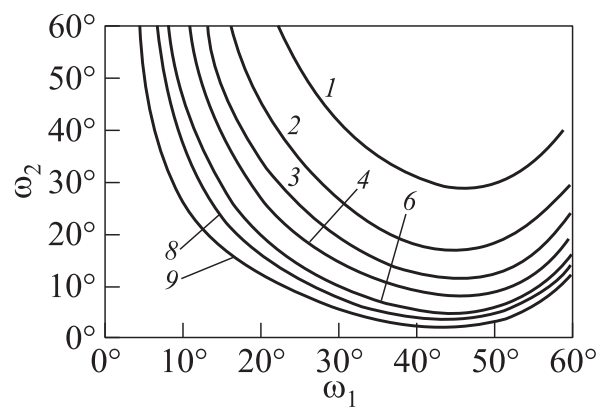

(a)

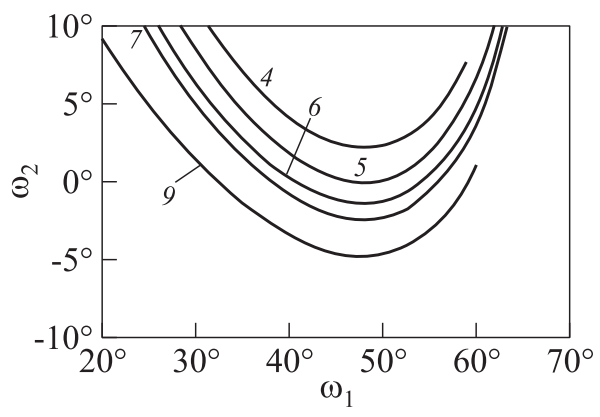

(b)

Figure 4 Dependence of the reflection angle $\omega_{2}$ on the angle of incidence $\omega_{1}$ for different Mach numbers $\mathrm{M}_{1}\left(1-\mathrm{M}_{1}=3 ; 2-4 ; 3-5 ; 4-6 ; 5-7 ; 6-8 ; 7-\right.$ $9 ; 8-10$; and $\left.9-\mathrm{M}_{1}=15\right)$ at a constant value $\gamma=1.4(a)$ and $1.3(b)$

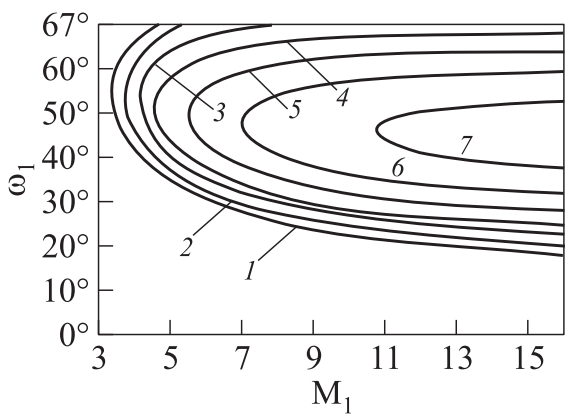

Figure 5 The boundaries of the existence of a three-shock configuration with the negative angle of reflection in the plane $\left(\mathrm{M}_{1}-\omega_{1}\right)$ in dependence on $\gamma: 1-\gamma=1.05$; $2-1.1 ; 3-1.15 ; 4-1.2 ; 5-1.25 ; 6-1.3$; and $7-1.35$. The angle $\omega_{2}$ becomes negative for the values of the parameters that are inside the corresponding curves

begin to shift down and part of the curves at high Mach numbers occur in the negative area. This is due to the fact that with decreasing $\gamma$, compressibility of gas increases and the deviation of the flow also increases. The deviation of the flow increases also with increasing Mach number. The resulting graph of the boundaries of the existence of the configuration with a negative angle $\omega_{2}=0$ is shown in Fig. 5 in the coordinates of $\omega_{1}-\mathrm{M}_{1}$ for different values of the adiabatic index $\gamma$. Negative angle appears inside the curves.

Thus, in answer to the first question, the boundaries and regions of existence of the new configuration have been found. The new configuration occurs when Mach number of flow $\mathrm{M}_{1}$ is greater than 3 and the adiabatic index $\gamma$ is less than 1.4. It should be noted that the first idea of the negative angles in 
three shock waves configurations due to the reduction of the adiabatic index in real gases has been developed in [11-13] in the study of reflection of shock waves in carbon dioxide, Freon-12, and dissociating air. Recently, the existence of a negative angle of reflection has been noted in $[14,15]$, when the existence and characteristics of shock wave triple points are examined for the adiabatic indexes $\gamma$ equal to $1,1.4$, and 1.66 and Mach numbers $\mathrm{M}_{1}$ ranging from solution onset to 6 . The appearance of a negative angle occurs in the range of Mach numbers 2.5-6 when $\gamma=1$. Thus, a very large impact has been revealed of the reality of gas on the irregular reflection of shock waves. In this regard, it is interesting to determine the influence of a change in the adiabatic index on the boundary of transition from regular to Mach reflection and to build a complete picture of the reflection of shock waves as a function of the adiabatic index.

\subsection{Complete Picture of the Types of Reflection as a Function of the Adiabatic Index}

Location of shock waves depends on three parameters: Mach number of free stream $\mathrm{M}_{1}$, incident angle $\omega_{1}$, and effective value of the adiabatic index $\gamma$. Figure 6 shows the well-known transition boundaries of the regular and irregular reflection depending on the Mach number $M_{1}$ and the angle of incidence $\omega_{1}$ with the effective value of the adiabatic index equal to 1.4 [16]. It is seen that there is a region of double solution where it is possible to get a two- and threeshock configurations. This fact explains the so-called hysteresis phenomenon. It turned out that the type of configuration depends on the transition to this area. At a very slow transition from the regular reflection, it is delayed and

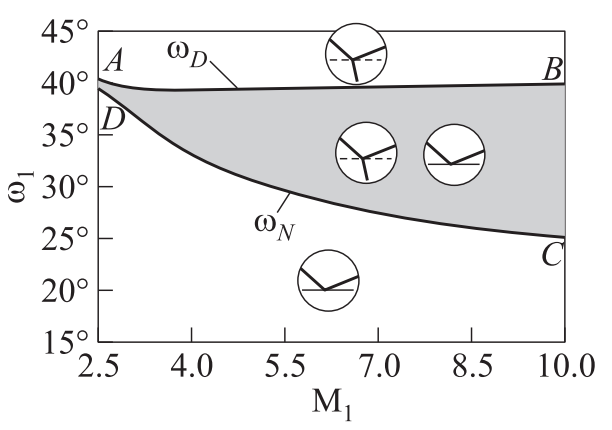

Figure 6 The curves of transition boundaries between Mach reflection and regular reflection $\omega_{D}$ and $\omega_{N}$ depending on the Mach number of the flow at the adiabatic index $\gamma=1.4 ; A B C D$ - domain of dual solution where both regular and irregular reflections are possible 


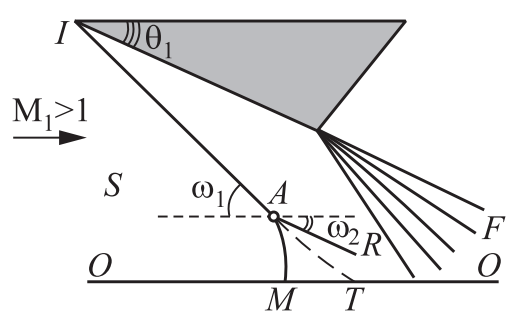

Figure 7 Three-shock configuration of shock waves with a negative angle of reflection $\omega_{2}<0$

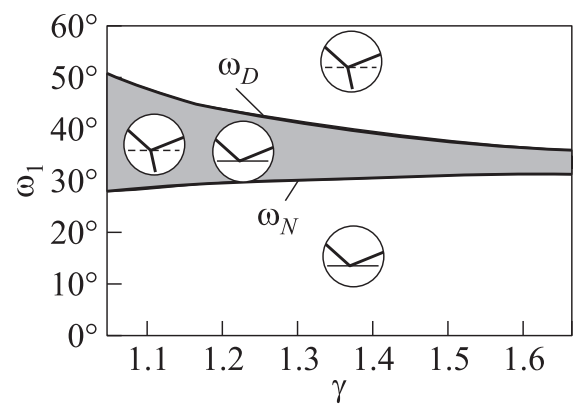

Figure 8 The curves of critical angles $\omega_{D}$ and $\omega_{N}$ in dependence on an adiabatic index $\gamma$ for flow Mach number $\mathrm{M}_{1}=5$. In the area $A B C D$, both regular and irregular reflections are possible

exists in this area up to the angle $\omega_{D}$. If one returns back from Mach reflection, then Mach reflection is delayed and the transition to regular reflection occurs at the von Neumann angle $\omega_{N}$. This conclusion follows from theoretical considerations and numerical experiments but in physical experiment, Mach reflection is more stable in the area of double solution than the regular one. Hysteresis phenomenon was studied in numerous works but only for a constant adiabatic index $\gamma=1.4[16-18]$.

Let find the dependence of boundaries between the regular and Mach reflection on the value of the adiabatic index and describe the general picture of all forms of reflection including the new form of reflection (Fig. 7).

Boundaries and area of regular and irregular reflection have been determined by shock polars method [10]. The calculation has been carried out for a range of Mach numbers from 1 to 15 , the incidence angles from $0^{\circ}$ to $90^{\circ}$, and the adiabatic index from 1.05 to 1.66. The transition angle $\omega_{D}$ for the regular reflection has been determined (at greater angles, regular reflection cannot exist) in dependence on the Mach number of free stream $\mathrm{M}_{1}$ and the adiabatic index $\gamma$. For irregular reflection, critical angle $\omega_{N}$ has been determined, below which the irregular reflection cannot exist. The region of dual solution lies between these two curves. It is known that the area of dual solutions for a constant adiabatic index $\gamma=1.4$ increases rapidly with increasing Mach number [18]. It has been shown that the same relationship exists for the other values of the adiabatic index. The effect of the adiabatic index (at constant Mach number) on the domain of dual solutions has been studied. The calculations show (Fig. 8) that with a decrease in the value of the adiabatic index, the region of double solution increases. For example, for a constant $\mathrm{M}_{1}=5$ and changes in $\gamma$ from 1.4 to 1.05, the domain of double solution increases by $14^{\circ}$. 


\subsection{New Areas of Dual Solution}

Let combine the boundaries of transition between regular and Mach reflections with the boundary of the existence of the new configuration with a negative angle of reflection $\omega_{G}$. Figure 9 shows the total picture of all types of reflection at the constant adiabatic index $\gamma=1.28$. It includes all three boundaries of existence: of regular reflection $(A B)$, of irregular $(D C)$, and of the configuration with a negative angle of reflection $(E G H)$. From this figure, one can see that except for the region of double solution $A G H C D$ where there are possible both Mach and regular reflections, a new region of double solutions $B G H$ has been found. In the region, a regular reflection configuration is possible as well as irregular with a negative angle of reflection. Note that when the adiabatic index is greater or equal to 1.4, there is only one area of dual solutions $A B C D$, as it was shown in Fig. 6, since at the adiabatic index greater or equal to 1.4, a configuration with a negative angle does not occur.

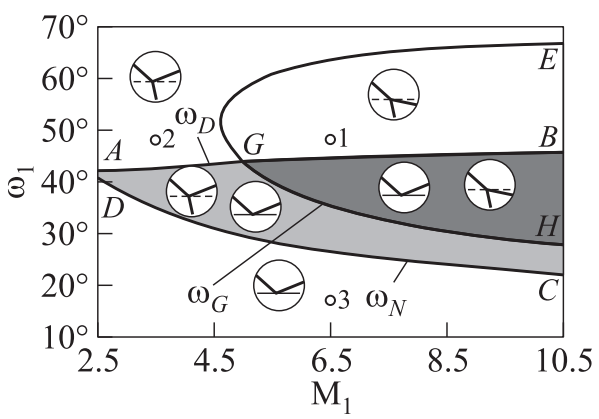

Figure 9 The curves of the transition angles $\omega_{N}, \omega_{D}$, and $\omega_{G}$ in dependence on the Mach number of flow for adiabatic index $\gamma=1.28$; AGHCD - domain of dual solution where both regular and irregular reflections may occur and $G B H$ - domain in which there are possible both regular and irregular reflections with a negative angle of reflection

\section{NUMERICAL STUDY}

\subsection{Description of Numerical Method of Calculation and Comparison with Experiments}

Analytical theory determines only the location of the shock waves in the close vicinity of the triple point. Therefore, a complete picture of the flow and behavior of configuration with negative angle has been investigated numerically. 


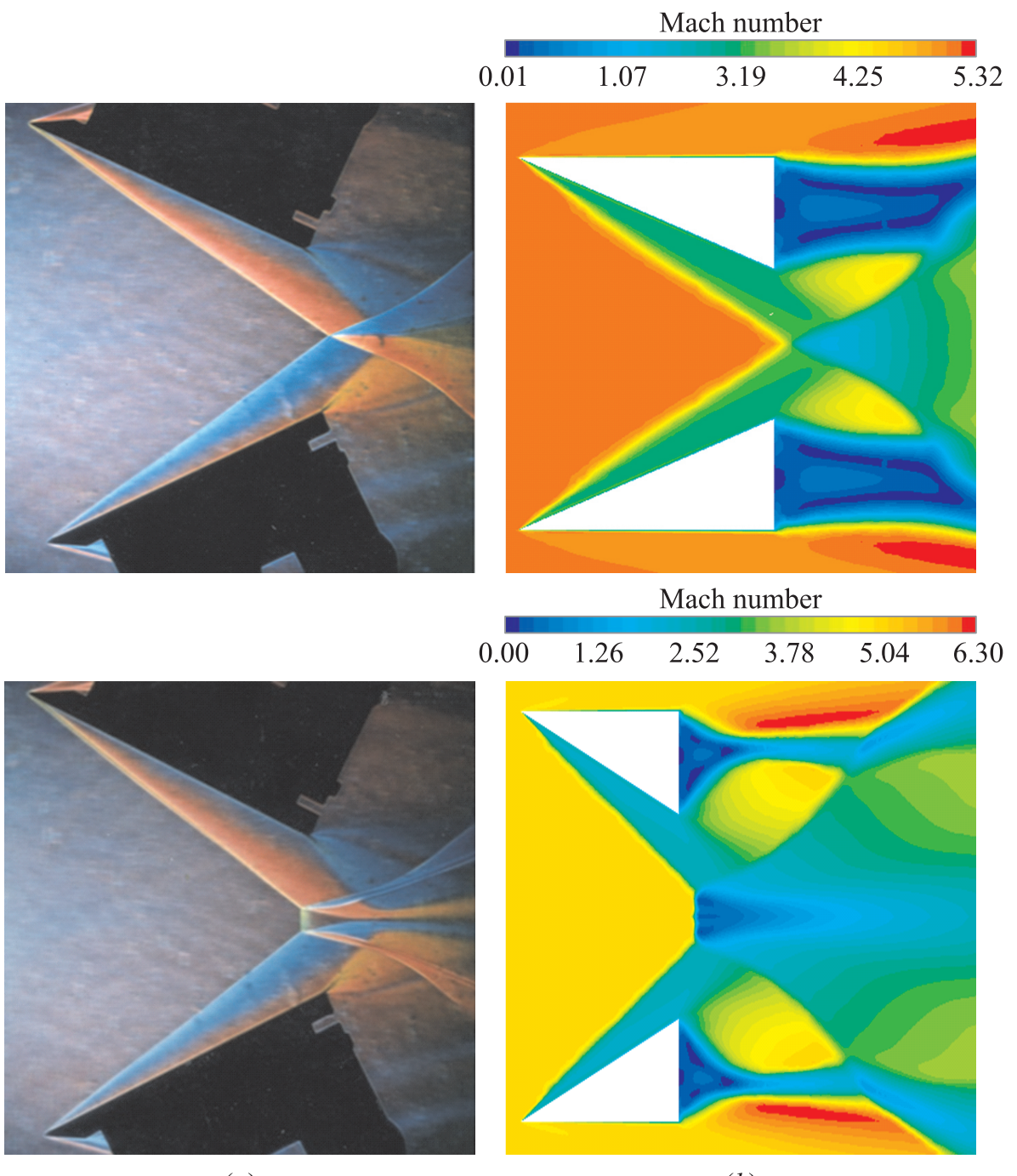

(a)

(b)

Figure 10 Comparison of the results of experiments in a wind tunnel [21] (a) and the numerical calculations $(b)$ for regular (upper row) and Mach (lower row) reflections. The flow parameters: $\mathrm{M}_{1}=4.95$ and $\gamma=1.4$

Computing program STAR-CCM + v. 6.06 has been applied for the numerical calculation. It is designed for solving the problems of continuum mechanics [19]. The program incorporates a method of averaging the Navier-Stokes equations (RANS - Reynolds-averaged Navier-Stokes). To close the system 
of equations due to the appearance of the new features, the model SpalartAllmaras has been applied to characterize the turbulent stresses [20]. Reflection of the shock wave from the plane of symmetry has been studied as a model. The shock wave was generated by a wedge placed in a supersonic flow (see Figs. $1 a$ and $1 b$ ). The calculations were carried out at different angles of wedges, $\theta_{1}$, varied from $10^{\circ}$ to $50^{\circ}$ and in a wide range of flow parameters: $\gamma=1.05-1.4$ and $\mathrm{M}_{1}=3-10$.

For the numerical calculation, a rectangular area was chosen which is extended in the horizontal plane with a wedge located at the left part of it. Due to the symmetry problem, the solution was carried out for only one-half of the geometry with a symmetry condition at the lower boundary. On the left boundary, the Mach number and the static pressure were set; at the top and the right boundary, only the static pressure was set. The calculation was performed on a multifaceted two-dimensional grid. On the wedge boundary, prismatic layers were built necessary for calculation of the turbulence.

Numerical calculations simulated the free flow of gas. It was assumed that the initial conditions coincide with the boundary ones. Inlet pressure was $P_{0}$ $=101325 \mathrm{~Pa}$ and temperature was $T_{0}=300 \mathrm{~K}$. The equation of state of an ideal gas was set. Courant number was set equal to 0.1 initially and was changed up or down depending on the convergence of the solution.

To test the program, a series of preliminary calculations for regular and Mach reflection for different angles of the wedge and the parameters of the flow have been made in those areas where only the configuration with a positive angle is obtained. Figure 10 shows the comparison between the results of numerical calculation and the experiment in wind tunnel for regular and irregular reflection.

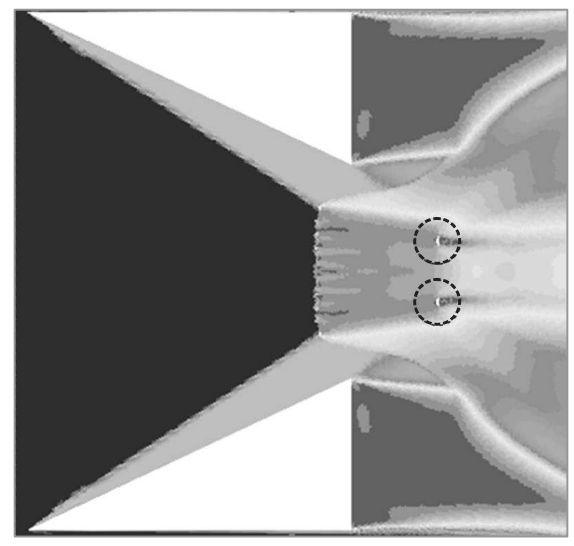

Figure 11 Result of the numerical calculation of Mach reflection with incorporated small wedges. The flow parameters: $\mathrm{M}_{1}=4.95$ and $\gamma=1.4$ 
A good visual agreement between the results is seen. Comparison of the gas parameters and location of the waves in the vicinity of the triple point $A$ also gave good agreement with analytic calculations. As it was noted, the three-shock theory does not determine the height of the Mach stem. It is determined by the geometry of the wedge and the distance between the wedges; so, in the figure, there is a slight difference in the value of height of the Mach stem.

The authors have suggested a method to control the height of Mach stem. A system of two small wedges was included downstream. As a result, the Mach stem began to move back upstream, as is seen in Fig. 11. Thus, it is possible to vary the height of the Mach stem by changing the size and location of the wedges in the flow.

\subsection{Configuration with a Kink on the Reflected Wave}

The focus of this paper is on the calculation inside the region of existence of a negative angle of reflection. Research in this area is very complicated because of the influence of the geometric characteristics of the downstream environment on the wave flow pattern. Analytical calculations cannot take this into account, they define the parameters of the flow behind shock waves only near the triple point.

First of all, the flow pattern can be affected by the rarefaction fan $S F$ emanating from the lower edge of the wedge (see Figs. $1 a$ and $1 b$ ). For example, at the Mach number $\mathrm{M}_{1}=6$ and the adiabatic index $\gamma=1.08$, the analytical calculations show that a three-shock configuration with a negative angle should arise. However, the rarefaction fan emanating from the lower edge of the wedge intersects the incident wave, the incident wave is curved, the angle of incidence decreases, and a complex stable stationary configuration, like regular one, was received which cannot be described with three-shock theory because the incident wave is curved (Fig. 12).

In order to produce a three-shock wave configuration with a negative angle of reflection, it was necessary to find the calculated geometry so that the rarefaction fan interacts only with the reflected wave. This has been done in two ways: in the first case, a small wedge was put behind the Mach stem to move it upstream; in the second case, by changing the distance between the bottom edge of the wedge and the line of symmetry. As a result, the irregular reflection pattern with a negative angle of reflection in steady supersonic flow has been obtained for the first time (Fig. 13).

As can be seen from the results of numerical experiments, the reflected angle $\omega_{2}=-6.7^{\circ}$ in full accordance with the analytical calculation, whose results are shown in Fig. 13b. The interaction of the reflected wave with the rarefaction fan led to the change in angle, its movement to a positive value. 


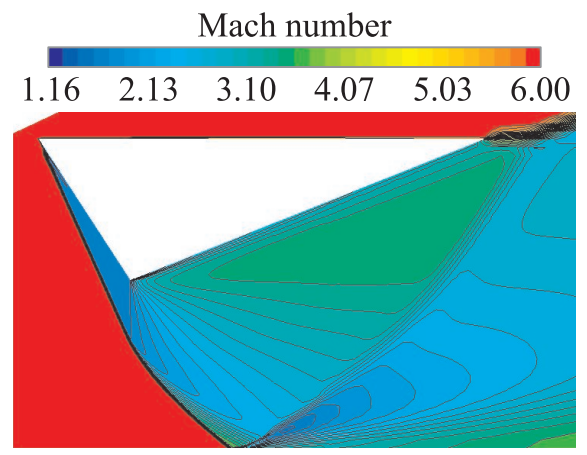

Figure 12 Changing of the shape of the incident wave due to the influence of the rarefaction fan. The flow parameters: $\mathrm{M}_{1}=6 ; \omega_{1}=65.9^{\circ} ; \theta_{1}=56.9^{\circ}$; and $\gamma=1.08$

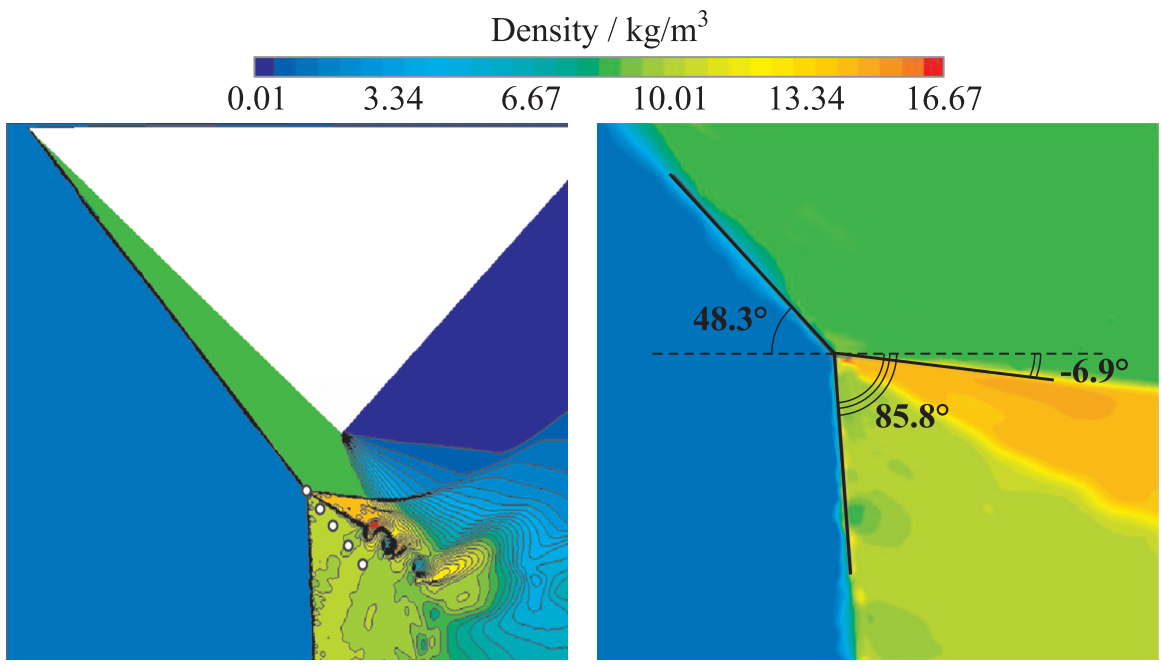

(a)

(b)

Figure 13 (a) Three-shock wave configuration with a negative angle of reflection and a kink in the reflected wave. Flow parameters: $\mathrm{M}_{1}=6.5 ; \omega_{1}=48.3^{\circ} ; \theta_{1}=40^{\circ}$; $\gamma=1.2$; and $\omega_{2}=-6.9^{\circ}$. The curvature of the reflected wave is due to the influence of the rarefaction fan. Open circles show the location of the sequence of position of the triple point. (b) Larger image of the triple point. The values of the angles are calculated with the three-shock theory 
However, despite the fact that the reflected wave has not crossed the line of symmetry, as shown in Fig. 2, the configuration is unstable. It arises in the process of setting and is not stationary. The triple point moves upstream. The intermediate position of the triple point is shown in Fig. 13a with open circles. The behavior of irregular reflection with the negative angle and a kink in the reflected wave needs further investigation. Schematic representation of the new configuration with a kink in the reflected wave is shown in Fig. 14.

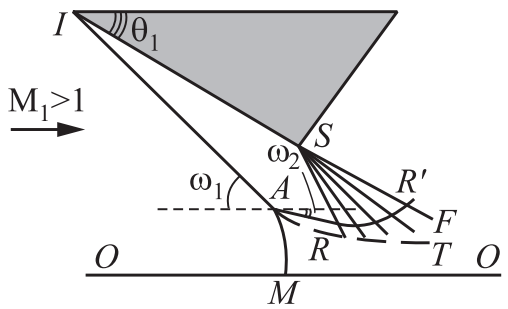

Figure 14 Schematic representation of the new configuration with a negative angle of reflection and a kink in the reflected wave

\subsection{Double Mach Reflection in Stationary Supersonic Gas Flow}

It has been found that there are several forms of reflection with a negative angle of reflection. Analytical calculations of three-shock configuration have provided only the location of shock waves in the immediate vicinity of the triple point, i.e., the initial part of the reflected wave near the triple point in the configuration with a negative angle of reflection should be directed downward relative to the flow (see Fig. $2 a$ ). General view of the resulting configuration and the height of Mach stem will depend on the geometry of the system of wedges and the other conditions downstream.

Numerical studies have shown that the forms of three-shock configuration with a negative angle of reflection will depend on the way of transition to this domain: from Mach reflection or from regular reflection. It has been found that if the transition from Mach reflection occurs, then irregular reflection with the negative angle and a kink in the reflected wave occur. If the transition from regular reflection takes place, then irregular reflection with the second triple point on the reflected wave occurs, i.e., double Mach reflection. The double Mach configuration is absolutely unstable. The triple point moves upstream.

Figures 13 and 15 show the results of calculations of three-shock configuration at the same geometry and the same flow parameters (Mach number $=6.5$, adiabatic index $=1.2$, and incident wave angle $\left.=48.3^{\circ}\right)$ in the domain of the existence of negative angle of reflection (point 1 in the domain $E G B$, see Fig. 9). Two completely different results have been obtained. One can come to the point 1 in two different ways, depending on the initial conditions. If one first gets stable configuration with a positive angle of reflection (point 2 in Fig. 9) and if one changes the conditions of the incoming flow so that a configuration 


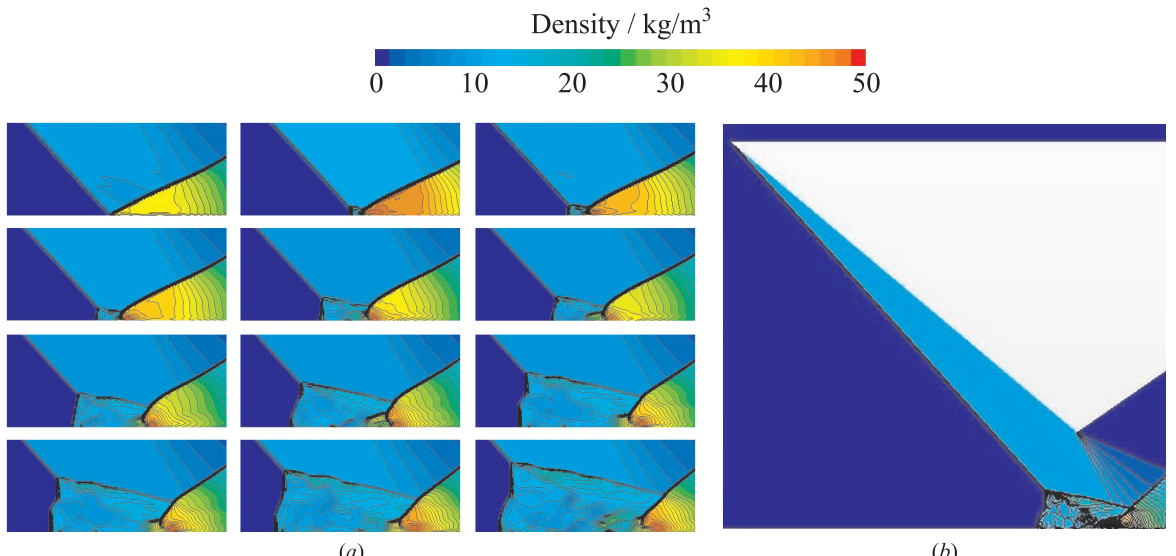

Figure 15 Sequence of pictures of the unstable double Mach reflection $(a)$ and the overall look $(b)$ : Mach number $\mathrm{M}_{1}=6.5$; adiabatic index $\gamma=1.2$; wedge angle $\theta_{1}=40^{\circ} ;$ and incident wave angle $\omega_{1}=48.3^{\circ}$

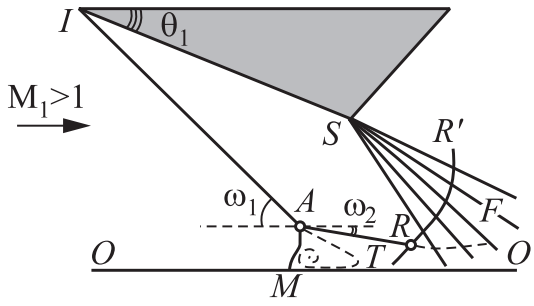

Figure 16 The scheme of DMR with a negative reflection angle with a negative angle of reflection occurs (point 1), then one gets a threeshock configuration with the kink in the reflected wave. In another case, if at the initial time of the calculation there is a regular reflection (point 3), then for rapid transition from regular reflection (changing the angle of the incident wave $\left.\omega_{1}\right)$ to configuration with a negative angle of reflection (from point 3 to point 1 ), one gets double Mach reflection (DMR).

Figure 13 shows the configuration with a negative angle of reflection and the kink in the reflected wave at the transition from Mach reflection with a positive angle of reflection to configuration with a negative angle of reflection. The dots mark the position of the triple point. The triple point moves upstream.

Figure 15 shows the result of the transition from regular reflection to a configuration with a negative angle of reflection. As one can see, there is a DMR which leads to disruption of steady-state flow.

Figure 16 gives the scheme of double Mach reflection with a negative reflection angle. Double Mach reflection was observed previously only in quasi-steady reflection from plane wedges. It is noted that this kind of reflection does not exist in stationary gas flows [16]. 


\section{CONCLUDING REMARKS}

A new configuration of shock waves has been found in the reflection of shock waves in a stationary supersonic gas flow in addition to the well-known regular and Mach reflections. This new three-shock configuration occurs with a negative angle of reflection and Mach numbers greater than 3 and an adiabatic index smaller than 1.4. Boundaries and areas of existence of this new configuration have been analytically defined.

The complete picture of the different types of reflections is given depending on the value of the adiabatic index. It has been shown that the domain formerly known as the domain of dual solution, where there are regular and Mach reflections, increases with the adiabatic index decrease. It was also found that at Mach numbers greater than 3 and the adiabatic index less than 1.4, there is another domain of dual solution. In this domain, either the regular reflection or configurations with a negative reflection angle are possible.

It has been shown that the configuration with a negative angle of reflection may take different forms depending on the transition. In the transition from Mach reflection, a three-shock configuration has a kink in the reflected wave. The reflection with a second triple point on the reflected wave (DMR) appears in the transition from regular reflection. The behavior of both configurations needs further investigation.

The appearance of new configurations may lead to the disruption of the stationary flow pattern. This result may be useful in predicting emergency situations in aircraft flight and rocket engine operation.

\section{ACKNOWLEDGMENTS}

The present study is supported by the Russian Foundation for Basic Research, grants Nos. 12-01-31362 and 14-08-01070.

\section{REFERENCES}

1. Courant, R., and K. O. Friedrichs. 1948. Supersonic flows and shock waves. New York: Intersicence. $464 \mathrm{p}$.

2. Landau, L. D., and E. M. Lifshitz. 1987. Fluid mechanics. 2nd ed. ButterworthHeineman: Linacre House; Oxford: Jordan Hill. Vol. 6. 551 p. (Translated from Russian by J. B. Sykes and W. H. Reid.)

3. Gvozdeva, L. G. 2010. Conditions of instability of three shock configuration in steady flows. ISIS-19. Moscow.

4. Gavrenkov, S. A., and L. G. Gvozdeva. 2011. Numeral investigation of triple shock configuration for steady cases in real gases. Physics of Extreme States of Matter Proceedings. Chernogolovka. 66-68. 
5. Gvozdeva, L. G., V. L. Borsch, and S. A. Gavrenkov. 2012. Analytical and numerical study of three shock configurations with negative reflection angle. 28th ISSW Proceedings. Ed. K. Kontis. Springer. 2:587-592.

6. Gvozdeva, L. G., and S. A. Gavrenkov. 2012. Formation of triple shock configurations with negative reflection angle in steady flows. Tech. Phys. Lett. 38(4):372-374.

7. Gvozdeva, L. G., and S. A. Gavrenkov. 2012. Numerical investigation of the onset of instability of triple shock configurations in steady supersonic gas flows. Tech. Phys. Lett. 38(6):587-589.

8. Gvozdeva, L. G., and S. A. Gavrenkov. 2012. Analytical determination of boundaries of existence of triple shock wave configuration with a negative angle of reflection in steady supersonic flow. Physics of Extreme States of Matter. Chernogolovka.

9. Gavrenkov, S. A., and L. G. Gvozdeva. 2012. A new type of triple configuration in a steady supersonic flow. Physics of Extreme States of Matter. Chernogolovka.

10. Von Neumann, J. 1963. Collection of works. Oxford: Pergamon Press. 6:239-299.

11. Bazhenova, T. V., L. G. Gvozdeva, Y. S. Lobastov, I. M. Naboko, R. G. Nemkov, and O. A. Predvoditeleva. 1968. Shock waves in real gases. Moscow: Nauka. 198 p. [In Russian.] (English translation: Tech. Rep. TT-F-58, NASA Technical Translation.)

12. Bazhenova, T. V., L. G. Gvozdeva. 1977. Unsteady interaction of shock waves. Moscow: Nauka. 274 p. [In Russian.]

13. Bazhenova, T. V., L. G. Gvozdeva, and M. A. Nettleton. 1984. Unsteady interaction of shock waves. Prog. Aerosp. Sci. 21:249.

14. Emanuel, G. 2011. Shock wave triple-point morphology. Shock Waves 6:511-521.

15. Emanuel, G. 2012. Shock wave dynamics: Derivatives and related topics. Boca Raton, FL: CRC Press. 235 p.

16. Ben-Dor, G. 2007. Shock wave reflection phenomena. 2nd ed. New York: SpringerVerlag. $356 \mathrm{p}$.

17. Hornung, H. G., and M. L. Robinson. 1982. Transition from regular to Mach reflection of shock wave. Part 2. The steady-flow criterion. J. Fluid Mech. 123:155-164.

18. Ivanov, M. S., S.F. Gimelshein, and A. E. Beylich. 1995. Hysteresis effect in stationary reflection of shock waves. Phys. Fluids 7:685-687.

19. STAR-CCM + Version 6.06 User's Manual. 2011. CD-Adapco.

20. Spalart, P. R., and S. R. Allmaras, 1992. A one-equation turbulence model for aerodynamic flows. AIAA Paper No. 92-0439

21. Passerel, D. 1996. Etude de la transition entre reflexion reguliere et reflexion de Mach. These de doctorat de l'Universite Pierre et Marie Curie. Paris. 157 p. 